\title{
Impact behaviour of preloaded glass/polyester woven plates
}

\author{
S.K. García-Castillo, S. Sánchez-Sáez, J. López-Puente, E. Barbero, C. Navarro* \\ Department of Continuum Mechanics and Structural Analysis, University Carlos III of Madrid, Avda. de la Universidad 30, 28911 Leganés, Madrid, Spain
}

\begin{abstract}
In this work, the effect of a biaxial preload in the behaviour of glass/polyester woven laminate plates subjected to high velocity trans versal impact was studied. For this, an analytic model based on energy considerations that include the presence of an in plane preload was used. The results of the analytic model for the biaxial preload state were compared with those found for a non preload plate, the difference between them being minimal for the pre stressed level reached in the tests ( $31 \%$ of the static UTS). Therefore, numerical simulations were made in order to study the effect of the preload in greater detail; furthermore, experimental tests were conducted, validating the analytic and numerical model. In general, the two methods revealed minimal differences between the values of the ballistic limit and those of the residual velocity.
\end{abstract}

Keywords: A. Glass fibres; A. Composite materials; B. Impact behaviour; C. Modelling; In plane preloaded

\section{Introduction}

Glass/polyester composites are being widely used in the transport industry to manufacture structural components that can be subjected to impulsive loads during their lifetime of service or during operations of assembly and maintenance. Therefore, it is necessary to determine their behaviour under these loads, given their greater sensitivity to impact damage than metals [1]. Two types of impact are usually considered, low- and high-velocity impact. The first type can produce internal defects in the form of delaminations that significantly reduce the residual strength and stiffness of the structure. High-velocity impact can perforate the structure, compromising its structural integrity. Several studies examine the behaviour of structural elements subjected to low-velocity impact [2,3] and highvelocity impact $[4,5]$.

Most studies on impact focus on load-free plates; however, in many cases the structural elements can be submitted to in-plane loads. A relatively small number of studies

\footnotetext{
* Corresponding author. Tel.: +349162494 91; fax: +34916249430.

E mail address: navarro@ing.uc3m.es (C. Navarro).
}

have considered the impact behaviour of uniaxially preloaded plates under tension [1,4,6,7] and compression [8]. However, this preloading condition does not properly reproduce the complex stress-state that appears in practical structural problems. A few more realistic tests have been made in which the plates are biaxially preloaded, although most of the available literature concentrates on laminates subjected to low-velocity impact [9 12], and much less information is available on laminates under high-velocity impact. Whittingham et al. [11] affirm that, in carbon-fibre laminated plates with biaxial preload, the penetration depth, peak load, and absorbed energy are independent of the preload at low-impact energy. Khalili et al. [12] also state that in reinforced graphite/epoxy composites the influence of the uniaxial preload in the contact force is marginal; nevertheless, the deflection and contact duration are influenced significantly by the preload. However, Nettles et al. [6] indicate that in carbon/epoxy composites, when the tensile preload augments, the peak load increases and the compression after impact strength diminishes. Chiu et al. [7] observed that a tensile preload enlarges the damage area when the energy of the impact surpasses a certain value for quasi-isotropic graphite/epoxy laminates. 
A useful technique to study this behaviour is first to use an analytical model that provides approximate solutions and allows the understanding of the influence of different parameters that control the impact process. In the case of composite plates without preload, a great number of studies present analytic models to evaluate the behaviour of such materials under impact loads both at low velocity $[13,14]$ as well as high [15 18]. Many of the models used for high-velocity impact are based on energy considerations. Naik et al. [15] proposed an energy model based on that of Moyre et al. [16] to analyze the behaviour of woven laminates that take into account different energyabsorption mechanisms: cone formation, tensile failure of primary yarns, deformation of secondary yarns, delamination, matrix cracking, shear plugging, and friction during penetration. The main difference between the two models is the greater number of absorption mechanisms incorporated in the model of Naik et al. [15]. Lopez-Puente et al. [17] used an energy model in the study of carbon/epoxy laminates subjected to a high-velocity impact, which also considers different contributions to the energy absorbed by the panel. It differs from the previous ones in using distance instead of time as an integration variable. Other models are based on considering the deformation that results in the panel due to the formation of a cone, such as that proposed by Vinson and Walter [18]. These authors studied a carbon-fibre weave using a model based on conical-shell theory and geometric considerations to predict the deformation of the panel and the displacement of the cone formed.

Several authors have used analytical models to study preloaded plates subjected to impact, but only for the case of low-velocity impact. Sun and Chattopadhyay [19] analysed the behaviour of cross-ply laminate plates under initial biaxial stress when submitted to an impulsive low-velocity load, using a nonlinear analytic model that combines the Hertz contact-force law with plate theory. A similar approach was used by Khalili et al. [12] to analyse the same type of impact of reinforced graphite/epoxy plates subjected to uniaxial and biaxial tensile pre-stresses. The modelling of the behaviour against high-velocity impact of the preloaded plates is a complex matter, for which no references involving analytical models were found in the literature.

Nevertheless, numerical simulations are needed to achieve more accurate results in order to minimize the number of experimental tests and hence to reduce design costs. These models have to be assessed through experimental tests. As in analytical models, the theoretical studies of the preloaded plates subjected to impact concentrate on low-velocity impact. Kelkar et al. [1] analyze the behaviour against impact on carbon/epoxy plates employing a model of finite elements using the Tsai-Wu criterion to predict the failure of the panel. Zhang et al. [8] studied carbon/epoxy plates subjected to in-plane compressive load followed by low-velocity impact using a FEM code. They used the damage model of Chang and Chang [20] to predict the failure of the panel during impact.
In this study the influence of static tensile biaxial preload on the behaviour of plates under high-velocity impact load was examined. An analytical model was used to estimate the ballistic limit and residual velocity in preloaded plates. In addition, a numerical model has been used, using the criterion of Hou et al. [21] to predict the progressive failure of the panel. The results of the models were compared to those resulting from high-velocity impact tests on glass/polyester plain woven plates with a biaxial static preload, at velocities between 140 and $525 \mathrm{~m} / \mathrm{s}$.

\section{Analytical model}

\subsection{Model description}

For the present study an analytical model was developed, based on the models of Naik et al. [15], Moyre et al. [16] and Vinson and Walter [18], while adding the effect of an in-plane preloading. This model allows an estimate of the residual velocity of the projectile (hence the ballistic limit) and the energy absorbed during the penetration of the laminated plate $\left(E_{\mathrm{AB}}\right)$. The following hypotheses were considered:

The projectile is perfectly rigid and remains totally indeformable over impact.

The energies absorbed by shear plugging and friction are considered negligible [15]

The energies absorbed by tensile failure of primary yarns and deformation of secondary yarn are treated independently (Fig. 1).

Longitudinal and transversal wave velocities are the same in each lamina.

The velocity of projectile remains constant in each time integration interval $(\Delta t)$.

A moving cone is formed on the back side of the plate (Fig. 1).

The total energy in an instant of time $t_{i}$ can be defined as:

$E_{T_{i}}=E_{\mathrm{AB}_{i}}+E_{\mathrm{KE}_{i}}$

$E_{\mathrm{AB} i}$ is the energy absorbed during the penetration of the laminated plate (Eq. (2)), and $E_{\mathrm{KE} i}$ is the kinetic energy (Eq. (3)) at an instant of time $t_{i}$.

For the calculation of $E_{\mathrm{AB} i}$, Moyre et al. [16] proposed two energy-absorption mechanisms: tensile failure of the primary yarns $\left(E_{\mathrm{TF} i}\right)$, and elastic deformation of the secondary yarns $\left(E_{\mathrm{ED} i}\right)$. In addition to these, two new terms were added to improve the model accuracy: delamination damage $\left(E_{\mathrm{DL} i}\right)$ and matrix cracking $\left(E_{\mathrm{MC} i}\right)$, which were proposed by Naik et al. [15].

$E_{\mathrm{AB}_{i}}=E_{\mathrm{TF}_{i}}+E_{\mathrm{ED}_{i}}+E_{\mathrm{DL}_{i}}+E_{\mathrm{MC}_{i}}$

The kinetic energy $\left(E_{\mathrm{KE} i}\right)$ in an instant of time $\mathrm{t}_{i}$ is composed of two terms: the kinetic energy of the projectile of mass $m$ that moves at a velocity $v_{i}$, and the kinetic of the 


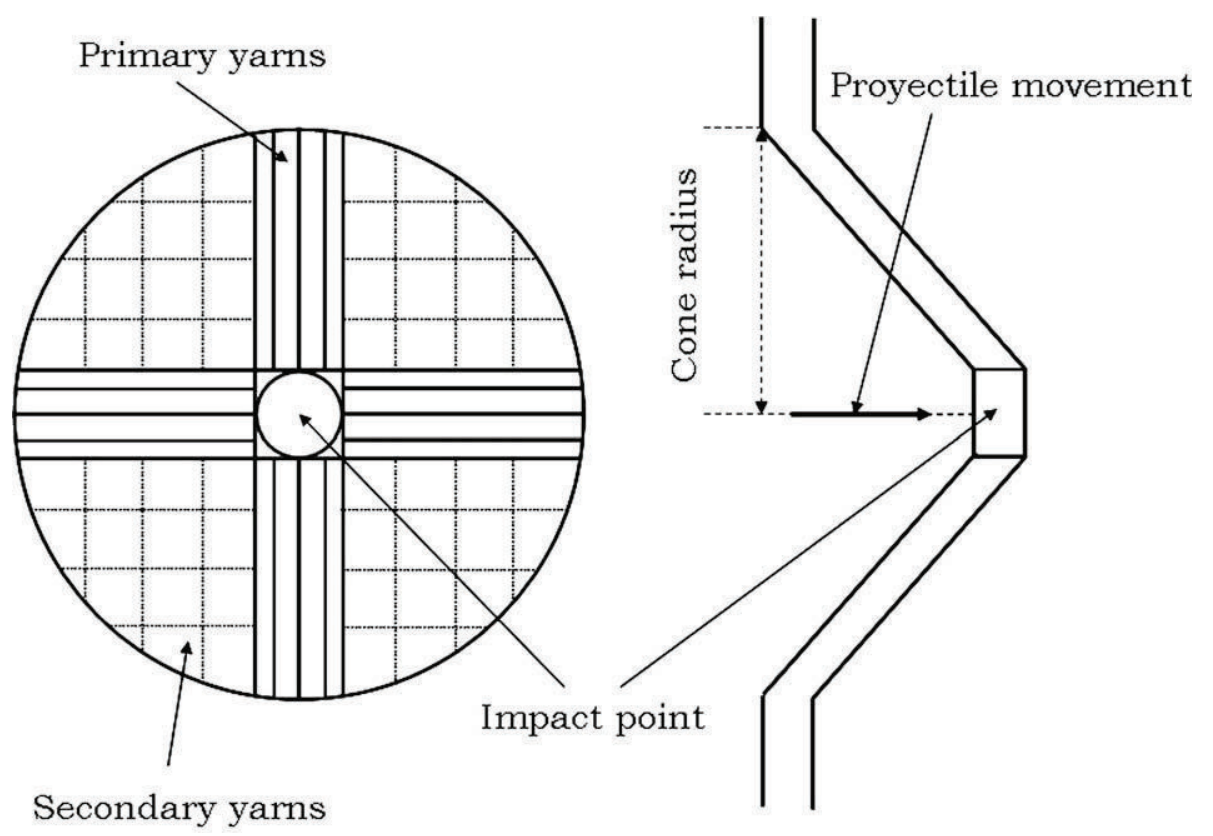

Fig. 1. Location of the primary and secondary yarns, and the cone formed in the plate.

moving cone formed on the back side of the plate of mass $\mathrm{M}_{\mathrm{c} i}$. It is assumed that the cone moves at the same velocity as the projectile. This energy is shown in Eq. (3).

$E_{\mathrm{KE}_{i}}=\frac{1}{2} \cdot\left(m+M_{\mathrm{c} i}\right) \cdot v_{i}^{2}$

The mass of the cone formed was defined by $M_{\mathrm{c}_{i}}=\pi \cdot R_{\mathrm{c}_{i}}^{2} \cdot e \cdot \rho$

where $e$ is the thickness of the target, and $R_{\mathrm{c} i}$ is radius of the cone formed on the back side of the plate for each instant of time. This radius was calculated by the Eq. (5):

$R_{\mathrm{c}_{i}}=v_{\mathrm{t}} \cdot t_{i}$

where $v_{\mathrm{t}}$ is the velocity of the transverse elastic waves generated by the impact on the plate, Eq. (6):

$v_{\mathrm{t}}=\sqrt{ } G_{23} / \rho$

where $G_{23}$ is the transverse shear modulus, and $\rho$ is the density of composite.

The total energy must be equal to the initial energy:

$E_{\mathrm{T}_{i}}=E_{\mathrm{AB}_{i}}+E_{\mathrm{KE}_{i}}=E_{\mathrm{KE}_{0}}$

Therefore the velocity of the projectile for each instant of time $\left(v_{i}\right)$ is:

$v_{i}=\sqrt{\frac{\frac{1}{2} \cdot m \cdot v_{0}^{2}-E_{\mathrm{AB}_{i}}}{\frac{1}{2} \cdot\left(m+M_{\mathrm{c}_{i}}\right)}}$

The absorbed energy can be rewritten as:

$E_{\mathrm{AB}_{i}}=\Delta E_{\mathrm{AB}_{i}}+E_{\mathrm{AB}_{i} 1}$

where $\Delta E_{\mathrm{AB}_{i}}$ is the absorbed energy in the interval of time between $t_{i} 1$ and $t_{i}$. For the calculation of $v_{i}$ it is assumed that $\Delta E_{\mathrm{AB}_{i}}$ is negligible.
If the projectile velocity is known in each instant of time $\left(t_{i}\right)$, then the deceleration of projectile may be calculated as:

$a_{i}=\frac{v_{i-1}-v_{i}}{\Delta t}$

Also, the distance travelled by the projectile $\left(Z_{i}\right)$ or the depth of the cone can be calculated with the following equation:

$Z_{i}=\sum_{i=0}^{i}\left(v_{i-1} \cdot \Delta t-\frac{1}{2} \cdot a_{i} \cdot(\Delta t)^{2}\right)$

And the strain in the primary yarns was calculated with this equation:

$\varepsilon_{i}=\frac{Z_{i} \cdot \sin \left(\theta_{i}\right) \cdot \cos \left(\theta_{i}\right)}{R_{\mathrm{p}_{i}} \cdot \ln \left(\left(R_{\mathrm{c}_{i}}+R_{\mathrm{l}_{i}}\right) / R_{\mathrm{l}_{i}}\right)}$

where $R_{\mathrm{p} i}$ for this analytical model was assumed equal to radius of the projectile and

$\theta_{i}=\tan ^{-1}\left(R_{\mathrm{c}_{i}} / Z_{i}\right)$

$\mathrm{R}_{1 i}$ is the distance covered by the longitudinal wave generated by the impact on the plate

$R_{\mathrm{l}_{i}}=v_{1} \cdot t_{i}$

where $v_{1}$ is the velocity of longitudinal wave:

$v_{l}=\sqrt{ } \sigma / \rho$

and $\sigma$ is the failure stress of the composite.

\subsection{Energy-absorption mechanisms}

The energy generated by the impact is consumed in accelerating the cone formed on the plate and in a series of mechanisms that are described below. 


\subsubsection{Energy absorbed due to tensile failure of primary yarns}

Fibres that undergo direct impact from the projectile are known by the name of primary yarns (Fig. 1), which failed in tension on reaching the failure strain.

The energy absorbed by tensile failure of primary yarns is

$E_{\mathrm{TF} i}=c \cdot e \cdot \int_{0}^{R_{\mathrm{li}}}\left(\int_{\varepsilon_{\mathrm{in}}}^{\varepsilon_{\mathrm{r}} \cdot b^{(x / c)}} \sigma(\varepsilon) \cdot \mathrm{d} \varepsilon\right) \cdot \mathrm{d} x$

where $c$ is the yarn width, $\sigma(\varepsilon)$ is stress in function of the strain, $\varepsilon_{\text {in }}$ is initial strain (zero in the non-preload case), $\varepsilon_{\mathrm{r}}$ is the failure strain of the composite, and $b$ is the stress wave transmission factor.

\subsubsection{Energy absorbed due to deformation of secondary yarns}

The energy absorbed by elastic deformation of secondary yarns (Fig. 1) in the composite laminate was calculated from area under stress strain curve of material, which in this case presents linear behaviour.

The calculation of the energy absorbed by elastic deformation of secondary yarns was made using the following equation:

$E_{\mathrm{ED}_{i}}=4 \cdot E \cdot \varepsilon_{\mathrm{r}}^{2} \cdot e \cdot \pi \cdot \int_{D / 2}^{R_{\mathrm{ci}}}\left(\frac{R_{\mathrm{c}_{i}}-r}{2 \cdot R_{\mathrm{c}_{i}}-D}+\varepsilon_{\mathrm{in}}\right)^{2} \cdot r \cdot \mathrm{d} r$

where $E$ is the tensile modulus of the composite, $D$ is the diameter of the projectile and $r$ is the distance from the impact point, where $\varepsilon \quad \varepsilon_{\mathrm{r}}$ at $r \quad D / 2$ and $\varepsilon \quad 0$ at $r=R_{\mathrm{c}_{i}}$.

\subsubsection{Energy absorbed due to delamination and matrix cracking}

The formation of the cone on the back side of the plate produces two more damage mechanisms that contribute to the process of energy absorption. These damage mechanisms are called damage by delamination, and matrix cracking.

The damaged area that contributes to the energyabsorption process during the impact is confined to the cone radius, and thus for this analytic development, it was assumed that the area that contributes to the absorption mechanism by delamination and matrix cracking is given by the cone radius at each instant in time. Furthermore, the damaged area is estimated as a circular surface.

The energy absorbed due to delamination was calculated with the following equation:

$E_{\mathrm{DL}_{i}}=A \cdot R_{\mathrm{c}_{i}}^{2} \cdot G_{\mathrm{IIC}}$

where $A$ is the quasi-lemniscate reduction area and $G_{\text {IIC }}$ is the critical dynamic-strain energy-release rate in mode II.

The energy absorbed due matrix cracking was calculated as:

$E_{\mathrm{MC}_{i}}=A \cdot R_{\mathrm{c}_{i}}^{2} \cdot E_{\mathrm{MT}} \cdot e$

where $E_{M T}$ is the energy absorbed by matrix cracking per unit volume.

\section{Analytical results}

Particularizing the model for a glass/polyester woven laminate, the contribution of each energy-absorption
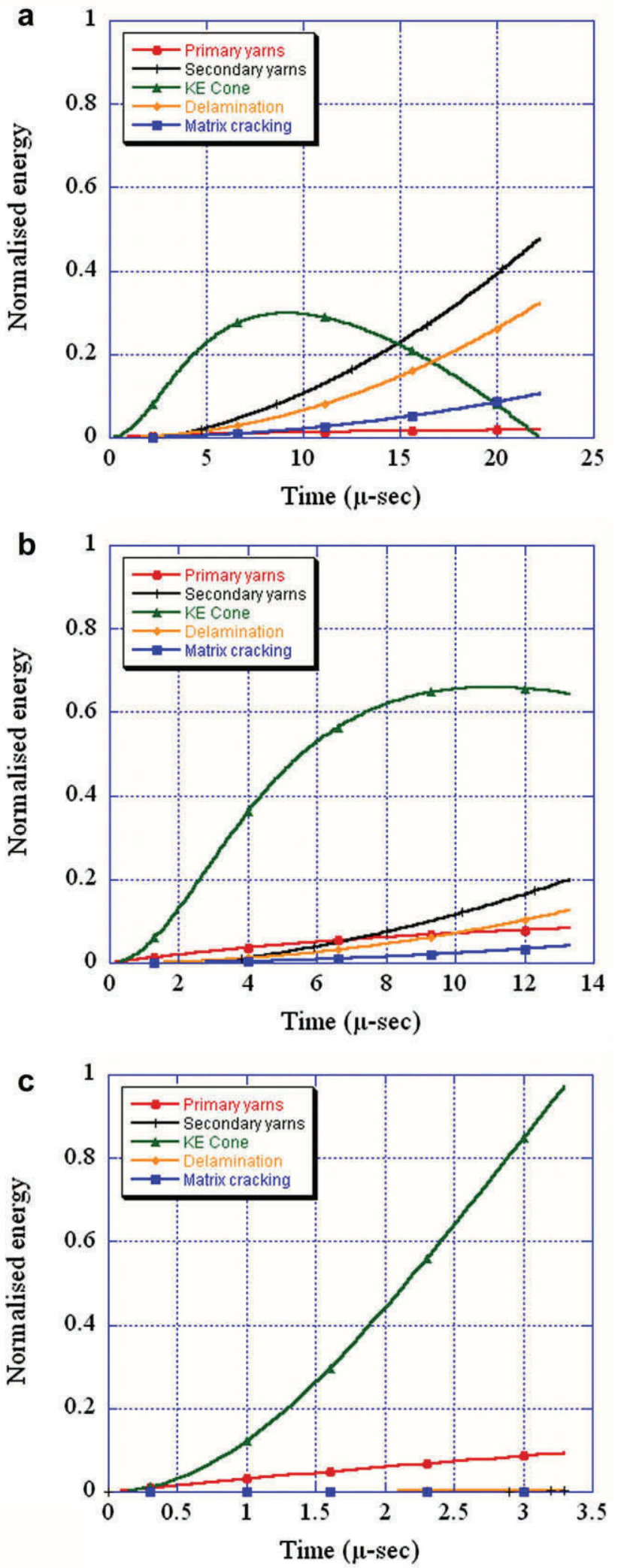

Fig. 2. Normalised energy versus time for non preloaded plates at an impact velocity of: (a) $163 \mathrm{~m} / \mathrm{s}$, (b). $216 \mathrm{~m} / \mathrm{s}$, (c) $512 \mathrm{~m} / \mathrm{s}$. 
Table 1

Ballistic limit

\begin{tabular}{lll}
\hline Ballistic limit $(\mathrm{m} / \mathrm{s})$ & Preloaded case & \\
\cline { 2 - 3 } & Non preloaded & Biaxially preloaded \\
\hline Experimental & 211 & 234 \\
Analytical & 209 & 215 \\
Numerical & 225 & 255 \\
\hline
\end{tabular}

mechanism was calculated from the energy absorbed due to each mechanism for non-preloaded and biaxial preloaded plates. The preload was $31 \%$ of the static UTS (367.4 MPa). Composite laminate properties were easily determined by conventional tests in the laboratory and by consulting the literature.

Fig. 2 presents the relationship of the normalised energy of each mechanism as a function of the time in the non-preloaded plates. The normalised energy is defined as the ratio between the energy absorbed by each mechanism and the total energy absorbed by the laminate.

Fig. 2a shows that the greatest contribution to the decrease in kinetic energy of the projectile corresponds to the energy absorbed due to deformation of secondary yarns and due to delamination, for impact velocities lower than the ballistic limit (Table 1). However, when the velocity is higher than the ballistic limit, Fig. $2 b$ and c, the formation of the cone on the back side of the plate becomes the main energy-absorption mechanism. It has been observed that neither the energy absorbed due to tensile failure of primary yarns nor matrix cracking contributes significantly to the energy absorbed by the plate.

The impact behaviour of the laminate described above does not seem to be affected as it is subjected to a biaxial pre-stressed state, as may be seen in Figs. 2 and 3.

\section{Numerical model}

The analytical model shows little influence of the in-plane load on the ballistic limit. In order to establish whether this influence is a true tendency, decreasing the residual velocity after impact, a numerical simulation with the finite-element method was made. In addition, the possibility of arranging a validated numerical code in order to simulate with accuracy the behaviour of composite laminates under ballistic impact is of great interest. For the numerical simulations, the finite-element commercial code ABAQUS was used; this software allows the creation of material models through user subroutines.

The material model used to describe the behaviour of woven glass/polyester laminates under ballistic impact derives from the Hou model [21], which was developed for carbon/epoxy tape laminates. Some modifications were required mainly due to the different reinforcement architecture. Only two of the four failure criteria were used:
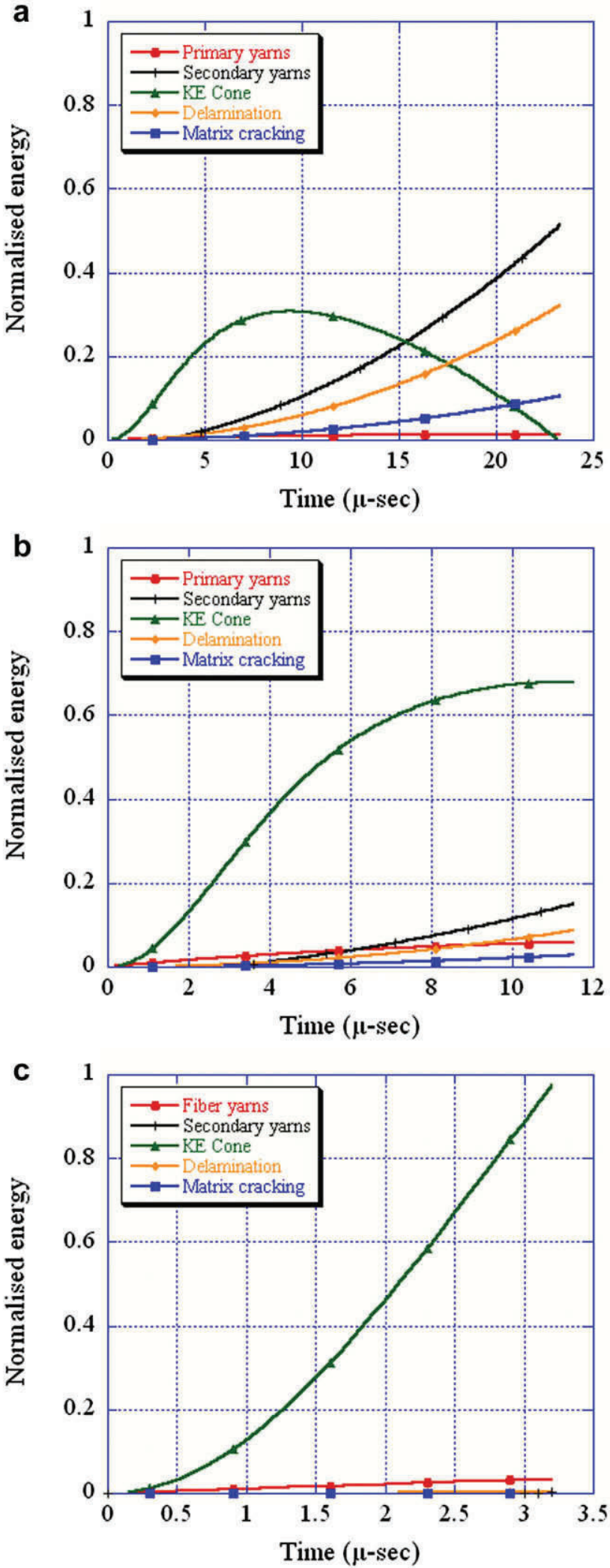

Fig. 3. Normalised energy versus time for biaxial preloaded plates at an impact velocity of: (a) $171 \mathrm{~m} / \mathrm{s}$, (b) $227 \mathrm{~m} / \mathrm{s}$, (c) $521 \mathrm{~m} / \mathrm{s}$.

Fibre failure; which describe both tensile and compression breakage; fibres run in two directions, hence two equations are used: for fibres at $0^{\circ}\left(d_{\mathrm{f} 1}\right)$ and for fibres at $90^{\circ}\left(d_{\mathrm{f} 2}\right)$. 
$d_{\mathrm{f}_{1}}=\left(\frac{\sigma_{1}}{X_{T}}\right)^{2}+\left(\frac{\tau_{12}^{2}+\tau_{13}^{2}}{S_{\mathrm{f}}^{2}}\right)$

$d_{\mathrm{f}_{2}}=\left(\frac{\sigma_{2}}{Y_{T}}\right)^{2}+\left(\frac{\tau_{12}^{2}+\tau_{23}^{2}}{S_{\mathrm{f}}^{2}}\right)$

where $\mathrm{X}_{T}$ and $\mathrm{Y}_{T}$ are the fibre strength properties in the 1 and 2 directions, respectively, and $\mathrm{S}_{\mathrm{f}}$ is through thickness shear strength of the fibre.

Delamination; the damage variable follows the equation

$d_{\mathrm{d}}=\left(\frac{\sigma_{3}}{Z_{\mathrm{r}}}\right)^{2}+\left(\frac{\tau_{23}}{S_{23}}\right)^{2}+\left(\frac{\tau_{13}}{S_{13}}\right)^{2}$

This criteria applies only to out-of-plane tension $\left(\sigma_{3}>0\right)$; $Z_{\mathrm{r}}$ represents the interlaminar strength, while $S_{23}$ and $S_{13}$ correspond to the shear strength in the 23 and 13 planes.

When the damage variable reaches the value of 1 , the material point is considered to have failed completely and hence for the rest of the simulation the stress components participating in the criterion are set to zero. For a smooth transition when the stress is set to zero, the following equation is used to correct the stress components:

$\sigma_{i j}^{\text {cor }}=\sigma_{i j}\left(1-\frac{2-\mathrm{e}^{s\left(d_{i}-1 / 2\right)}}{2-\mathrm{e}^{s / 2}}\right)$

where $s$ indicates the smoothness and $d_{i}$ is the damage parameter. An element erosion criterion was adopted in order to simulate the penetration of the laminate. This criterion eliminates the element reached.

The material model used for the projectile was linear elastic, because in the experimental test no plastic deformation was found in the tempered steel spheres after penetration. Eight-node hexahedral elements were used for the composite plate, whereas four-node tetrahedral elements were chosen for the steel sphere projectile.

\section{Experimental tests}

For experimental validation of the results of the analytical and numerical models, impact tests were made under in-plane tensile preloaded plates. The plates, with a thickness of $3.19 \mathrm{~mm}$, were made by five plies of E-glass/polyester plain weave.

To keep the specimens pre-loaded during the impact test, a special experimental device was designed and manufactured, and then it was coupled to a gas-cannon set-up. The device allows holding different static loads in two mutually orthogonal directions by two actuators. These actuators may work together or independently.

The tests made on plates with a static biaxial preload ( $31 \%$ of the static UTS in each direction), were compared with those made with non-preloaded plates. Cross-shape specimens $(200 \mathrm{~mm} \times 200 \mathrm{~mm})$ were used for the biaxial tests, while rectangular specimens $(140 \mathrm{~mm} \times 200 \mathrm{~mm})$ were used for the non-preload tests. The geometry and the shape of the first specimens were selected, after a fullnumerical simulation of the problem, in order to reach a uniform stress-state in the impacted zone.

The impact tests were made using a one-stage gas cannon manufactured by SABRE BALLISTIC. The specimens were impacted by steel spherical projectiles $7.5 \mathrm{~mm}$ in diameter, launched at velocities ranging from $140 \mathrm{~m} / \mathrm{s}$ to $525 \mathrm{~m} / \mathrm{s}$. During the impact tests, both the projectile striking velocity and the residual velocity were measured by a high-speed video camera PHOTRON FASTCAMultima APX.

\section{Results}

Fig. 4 presents the residual velocity as a function of the impact velocity for the analytic and numerical model and their validation with the experimental results. Both offer a good approximation to the experimental results. The differences between the models and the experimental results
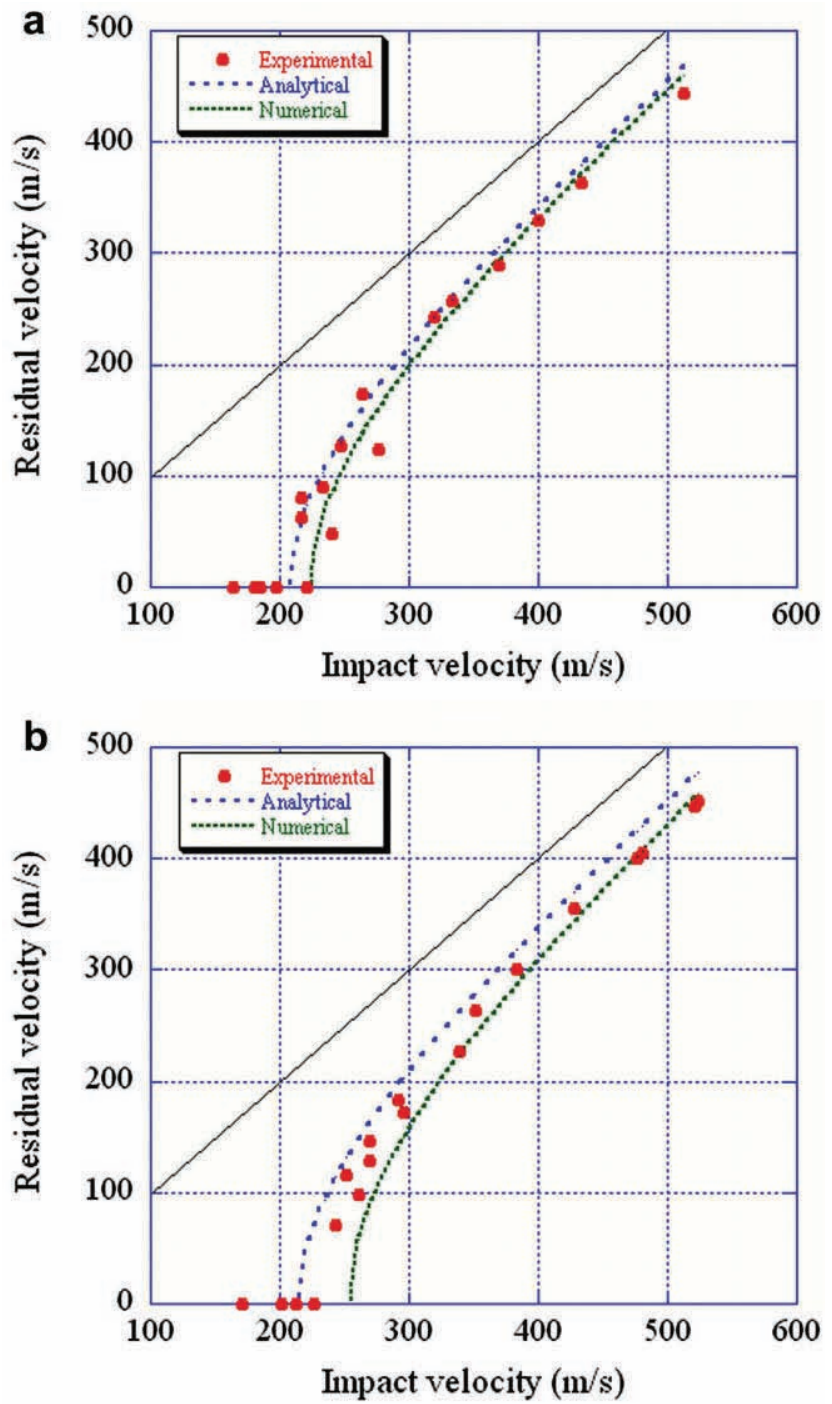

Fig. 4. Residual velocity vs. impact velocity in, (a) non preloaded plates, (b) biaxial preloaded plates. 
are somewhat higher in the case of the preloaded plates. Nevertheless, the differences between the residual-velocity curves for the non-preloaded and preloaded plates do not seem to be very high.

Also, the ballistic limit was determined from result of the analytic and numerical models (Table 1). The experimental ballistic limit was determined by the following equation $[22,23]$ :

$v_{\mathrm{r}}= \begin{cases}0, & 0<v_{o} \leqslant v_{\mathrm{L}} \\ B \cdot\left(v_{o}^{p}-v_{\mathrm{L}}^{p}\right)^{1 / p} & , v_{o}>v_{\mathrm{L}}\end{cases}$

where $v_{r}$ is the residual velocity, $v_{o}$ the impact velocity, $v_{L}$ the ballistic limit, and $p$ and $B$ are two empirical adjusting parameters.

It was observed that the preloading of the composite plates increased the ballistic limit. A good correlation was found between experimental, analytical and numerical results (Table 1), which demonstrate that the models used in this study faithfully reproduce the material behaviour.

\section{Conclusions}

The influence of the preload conditions (biaxial) on the behaviour of plates made of woven glass/polyester composite laminate materials under impact loading has been studied to determine the residual velocity and the ballistic limit.

In the biaxially preloaded plates, the ballistic limit for the projectile used proved approximately $11 \%$ higher. Both the analytic model and the numerical one reproduce this behaviour and can predict the ballistic limit for the nonpreloaded plates with a precision of $2 \%$ and with $8 \%$ in the case of the preloaded plates.

For velocities lower than the ballistic limit, the contribution of the total energy of failure of the secondary fibres was the greatest, while, for velocities far above the ballistic limit the greatest contribution was the formation of the cone. The existence of preload did not affect the contributions of each energy term.

The energy absorbed due to tensile failure of primary yarns and due to matrix cracking did not contribute significantly to the reduction of the kinetic energy of the projectile.

\section{Acknowledgements}

The authors are indebted to the Spanish Comisión Interministerial de Ciencia y Tecnología (Project MAT20010735) for the financial support of this work.

\section{References}

[1] Kelkar AD, Sankar J, Rajeev K. Analysis of tensile preloaded composites subjected to low velocity impact loads. AIAA J 1998;98(1944):1978 87.

[2] Cantwell WJ, Morton J. Geometrical effects in the low velocity impact response of CFRP. Compos Struct 1989;12(1):39 59.

[3] Richardson MOW, Wisheart MJ. Review of low velocity impact properties of composite materials. Compos Part A Appl Sci Manuf 1996;27(12):1123 31.

[4] Sankar BV, Sun CT. Low velocity impact damage in graphite epoxy laminates subjected to tensile initial stresses. AIAA J 1986;24(3):470 1.

[5] Nunes LM, Paciornik S. Evaluation of the damaged area of glass fiber reinforced epoxy matrix composite materials submitted to ballistic impacts. Compos Sci Technol 2004;64(7 8):945 54.

[6] Nettles A, Daniel V, Branscomb C. The effects of tensile preloads on the impact response of carbon/epoxy laminates. In: Proceedings of conference 40th international SAMPE symposium; 1995. p. 101925.

[7] Chiu S T, Liou Y Y, Chang Y C, Ong C L. Low velocity impact behaviour of prestressed composite laminates. Mater Chem Phys 1997;47:268 72 .

[8] Zhang X, Davies GAO, Hitchings D. Impact damage with compres sive preload and post impact compression of carbon composite plates. Int J Impact Eng 1999;22(5):485 509.

[9] Arnold WS, Robb MD, Marshall IH. Failure envelopes for notched CSM laminates under biaxial loading. Composites 1995;26:739 47.

[10] Robb MD, Arnold WS, Marshall IH. The damage tolerance of GRP laminates under biaxial prestress. Compos Struct 1995;32(1 4):141 9.

[11] Whittingham B, Marshall IH, Mitrevski T, Jones R. The response of composite structures with pre stress subject to low velocity impact damage. Compos Struct 2004;66(1 4):685 98.

[12] Khalili SMR, Mittal RK, Panah NM. Analysis of fiber reinforced composite plates subjected to transverse impact in the presence of initial stresses. Compos Struct 2007;77(2):263 8.

[13] Xion Y, Poon C, Straznicky PV, Vietinghoff H. A prediction method for the compressive strength of impact damaged composite laminates. Compos Struct 1995;30(4):357 67.

[14] Togh SL, Gong SW, Shim VPW. Transient stresses generated by low velocity impact on orthotropic laminated cylindrical shells. Compos Struct 1995;31(3):213 28.

[15] Naik NK, Shrirao P, Reddy BCK. Ballistic impact behaviour of woven fabric composites: formulation. Int J Impact Eng 2006;32(9): 1521 1552,.

[16] Moyre SS, Hine PJ, Duckett RA, Carr DJ, Ward IM. Modelling of the energy absorption by polymer composites upon ballistic impact. Compos Sci Technol 2000;60(14):2631 40.

[17] Lopez Puente J, Zaera R, Navarro C. High energy impact on woven laminates. J de Physique IV 2003;110:639 44.

[18] Vinson JR, Walter JM. Ballistic impact of thin walled composite structures. AIAA J 1997;35(5):875 8.

[19] Sun CT, Chattopadhyay S. Dynamic response of anisotropic lami nated plates under initial stress to impact of a mass. J Appl Mech 1975;42:693 8 .

[20] Chang FK, Chang KY. Post failure analysis of bolted composite joints in tension or shear out mode failure. J Compos Mater 1987;21(9):809 33

[21] Hou JP, Petrinic N, Ruiz C, Hallet SR. Prediction of impact damage in composite plates. Compos Sci Technol 2000;60(2):273 81.

[22] Zukas JA, Nicholas T, Swift H, Greszczuk LB, Curran DR. Impact Dynamic. Florida USA: Krieger Publishing Company; 1992.

[23] Kasano H. Recent advances in high velocity impact perforation of fiber composite laminates. JSME Int J, Serie A 1999;42(2):147 57. 